\title{
Maternal infections with an increased risk of transmission to the foetus; a literature review

\author{
Oana-Denisa Bălălău ${ }^{1,2}$, Octavian-Gabriel Olaru ${ }^{1,2}$, Adrian V. Dumitru ${ }^{1,3}$, Ioana \\ Păunică ${ }^{4}$, Anca Daniela Stănescu ${ }^{1,2}$ \\ ${ }^{1}$ CAROL DAVILA UNIVERSITY OF MEDICINE AND PHARMACY, BUCHAREST, ROMANIA \\ ${ }^{2}$ BUCUR MATERNITY, ST. JOHN EMERGENCY HOSPITAL, BUCHAREST, ROMANIA \\ ${ }^{3}$ EMERGENCY UNIVERSITY HOSPITAL, BUCHAREST, ROMANIA \\ ${ }^{4}$ NATL INST DIABET NUTR AND METAB DIS. PROF NC PAULESCU, BUCHAREST, ROMANIA
}

\section{AB STRACT}

Screening testing for infectious diseases with vertical transmission, from mother to foetus, decreases the prevalence of foetal malformations. The most common infections with teratogenic potential are found in the TORCH test (Toxoplasma, Other infections, Rubella, Cytomegalovirus, Herpes simplex virus). Early identification of these pathogens results in better neonatal outcomes. Most foetuses with congenital toxoplasmosis are asymptomatic or have no apparent birth defect, while the primary infection of pregnancy with varicella-zoster virus (VZV) can lead to congenital foetal abnormalities with devastating consequences. Treponema pallidum can easily infect the placenta, with transplacental transmission to the foetus that can occur from about 9-10 weeks of gestation. Also, hepatitis B and C are the most common causes of chronic viral hepatitis in children and adults. In the case of HIV-positive pregnant women, the early association of ART, together with the choice of birth by scheduled caesarean section and the complete lack of breastfeeding determine expected neonatal results, the risk of vertical transmission decreasing up to 1 $2 \%$. Primary rubella infection during pregnancy can significantly affect foetal development, as does Herpes simplex virus (HSV) infection, but if it occurs as close as possible to birth, the disease may be self-limiting with mild forms. No teratogenic agent should be described qualitatively, as a teratogenic exposure includes not only the agent but also the dose and time of pregnancy when the exposure will occur. Screening tests are recommended to be performed both preconceptionally and during pregnancy.

\section{Introduction}

During pregnancy, the woman may contract multiple infections associated with foetal risk. Infections acquired in utero or during the birth process are major causes of foetal and neonatal mortality, contributing significantly to the early and subsequent morbidity of the child.

To reduce this risk, it is necessary a quarterly screening testing, or more often if the patient reported exposure to infection, for as many pathogens as possible. Depending on the time of infection and the type of microorganism involved, significant foetal malformations may result.

These abnormalities are mainly the result of the action of infectious, physical agents, metabolic conditions or chemicals, and can cause foetal death as well as physical, behavioral and intellectual deficits [1]. The most accepted infectious teratogens are usually defined as TORCH (toxoplasma, other infection, rubella, cytomegalovirus, herpes simplex).

\section{Discussions}

If a woman is infected prenatal, perinatal, peripartum or even postnatal, these pathogens cause birth defects. Brent RL. et al. conducted a study on the causes of birth defects and concluded that in most of the patients in the group the cause remained unknown; the rest was genetic involvement [2].

Chen G. et al. analysed the relationships between exposure to environmental contraceptive risk factors and the risk of common congenital structural defects and showed that exposure to toxic substances in the work environment was the highest risk factor. Genital tract 
infections that associate with high fever during the first trimester of pregnancy and the administration of potential teratogenic drugs, such as hypnotics and anticonvulsants, could significantly increase the risk of structural birth defects. The correlation between oral contraceptive intake within six months before pregnancy and the risk of birth defects had no statistical significance [3].

A major importance in detecting infections that can complicate pregnancy is to perform the screening tests.

TORCH profile - includes testing for:

- Toxoplasma gondii

- Others (chickenpox, syphilis, hepatitis and human immunodeficiency viruses)

- Rubella

- Cytomegalovirus (CMV)

- Herpes simplex virus (HSV)

Congenital toxoplasmosis is caused by the protozoan parasite Toxoplasma gondii. The primary infection during pregnancy can lead to congenital diseases. Most foetuses with congenital toxoplasmosis are asymptomatic or have no apparent birth defects. Although the subclinical disease is apparently asymptomatic, there may be fever, maculopapular rash, hepatosplenomegaly, microcephaly, convulsions, jaundice, thrombocytopenia, even generalized lymphadenopathy. The classic triad of congenital toxoplasmosis consists of chorioretinitis, hydrocephalus and intracranial calcifications. A fetus with hydrocephalus may cause a dystocia of vaginal birth, requiring caesarean section.

Children with subclinical congenital toxoplasmosis who do not receive treatment have an increased risk of long-term sequelae. The most common late manifestation is chorioretinitis, which can lead to vision loss, intellectual deficit (mental retardation), deafness, seizures and spasticity [4].

Package C. et al. evaluated the effect of screening on the diagnosis of congenital toxoplasmosis and the effectiveness of prophylaxis and treatment, thus supporting the recommendation of the Obstetricians and Gynaecologists Society of Canada not to perform routine screening in the Canadian population for Toxoplasma gondii due to low prevalence and will be reserved only for pregnant women thought to be exposed to the risk of primary infection with Toxoplasma gondii [5]. If acute Toxoplasma gondii infection is suspected, repeated testing should be performed within 2 to 3 weeks.

Amniocentesis is reserved for the identification of Toxoplasma gondii in amniotic fluid by polymerase chain reaction: if primary maternal infection is diagnosed, if serological testing cannot confirm or rule out acute infection or in the presence of abnormal ultrasound findings (intracranial calcification, hydrocephalus, microcephaly, hepatosplenomegaly or severe intrauterine growth restriction).

After an infection with Toxoplasma gondii, it is recommended to delay conception for 6 months.

Syphilis (spirochete Treponema Pallidum) present in pregnancy and not treated properly can cause congenital syphilis and induce premature birth [6]. Treponema pallidum easily infects the placenta, with transplacental transmission to the fetus that can occur from about 9-10 weeks of gestation, regardless of the stage of the maternal disease [2]. It is important that the manifestations of congenital infection are influenced by gestational age, maternal condition, maternal treatment and the immune response of the fetus. Foetal abnormalities are the result of a strong inflammatory response to T. pallidum.

After infection of the placenta, the transplacental passage of the spirochete to the foetal circulation leads to foetal liver infections and dysfunctions, followed by infection of the amniotic fluid, foetal haematological abnormalities (anaemia, thrombocytopenia), ascites, hydrops and foetal IgM production. Hepatomegaly is thought to be caused by inflammation, extramedullary haematopoiesis, and hepatic congestion [7].

Infection of the new-born at birth may result from contact with maternal secretions or spirochetes containing blood, which is why it would be preferable to consider the birth by caesarean section [8].

Congenital syphilis - occurs when the spirochete Treponema pallidum is transmitted from a pregnant woman to the fetus. It can result in foetal hydrops, premature birth with long-term associated morbidity, or mortality at birth. Because of this morbidity, great emphasis is placed on routine screening for syphilis in all pregnant women [9]. Many cases of congenital syphilis develop because the mother has not received prenatal care. Most new-borns with congenital syphilis are asymptomatic at birth. The common infection may occur in the fetus from birth or may occur later in childhood.

Cheng JQ. et al., in an over 3-years study, had 477,656 pregnant women tested for syphilis, of which $2208(0.5 \%)$ were confirmed positive. From 2003 to 2005, they collected epidemiological and treatment data from 2019 cases of positive syphilis. Of these, 1855 (91.9\%) of pregnant women received treatment. Of the 1,020 babies born, 92 (9.0\%) had congenital syphilis. If we exclude mothers who had children with positive syphilis without undergoing prenatal screening, the success rate of the project for mother-child transmission intervention was $99.1 \%$, thus demonstrating the importance of testing for pregnant women with syphilis [9].

Chickenpox virus (VZV) is one of eight herpes viruses. Contracted in childhood, it generally produces mild manifestations. In adults, it presents noisy symptoms with a risk of significant morbidity and mortality. 
Primary infection in pregnancy causes pneumonia with VZV with a very severe maternal prognosis, which can lead to congenital foetal abnormalities with devastating consequences [10]. Most cases of congenital chickenpox syndrome occur in new-borns whose mothers were infected between 8 and 20 weeks of gestation and have the following manifestations [11-14]:

- cutaneous scars, which can be depressed and pigmented in the dermatomeric distribution

- neurological abnormalities (mental retardation, microcephaly, hydrocephalus, convulsions, Horner syndrome)

- cortical atrophy and convulsions

- ocular abnormalities (optic nerve atrophy, cataract, chorioretinitis, microphthalmia, nystagmus)

- limb abnormalities (hypoplasia, atrophy, paresis)

- gastrointestinal abnormalities (gastroesophageal reflux, atresia or intestinal stenosis)

- low weight at birth.

Prenatal diagnosis - consists of PCR (polymerase chain reaction) testing of foetal blood or amniotic fluid for VZV DNA together with ultrasonography to detect foetal abnormalities [11, 15-16].

Depending on the maternal and foetal diseases diagnosed prenatally, it will be decided how to give birth with the lowest risk of worsening the symptoms. Sanchez MA et al. conducted a prospective cohort study between 1993 and 2006 to describe the prevalence of congenital chickenpox syndrome (CVS) in the population of likely infected pregnant women. Positive IgM cases were subjected to monthly ultrasounds and foetal blood (FB) sampling (including anti-VZV IgM and virus culture). The amniotic fluid sample for PCR was added to the diagnosis of positive IgM cases after 1997. The rate of foetal infection in this cohort was $0.8 \%$, but the best expected prevalence of CVS, according to authors findings, should be $0.39 \%$ among infected women [17].

Hepatitis B virus (HBV) infection during pregnancy is usually mild and is not associated with increased mortality or teratogenicity [18-19]. However, cases of increased incidence of low weight at birth a prematurity have been reported in mothers with acute HBV infection [20-21]. The infection rate of new-borns in HBV-positive mothers is $90 \%$ for new-borns who do not receive human immunoglobulin antihepatitis B and hepatitis B vaccination at birth [22]. Mother-to-child transmission can occur in the uterus, at birth, or after birth. However, most infections occur at birth, which is why a caesarean delivery is to be considered.

Worldwide, hepatitis B and C are the most common causes of chronic viral hepatitis in children and adults [23]. Thanks to hepatitis B vaccination programs, the hepatitis $C$ virus (HCV) has become the leading cause of chronic viral hepatitis in children, with vertical transmission being the main source of infection [24-27]. Vertical transmission refers to viral transmission from mother to child, during pregnancy, birth or the first 28 days of life [28]. Obstetric management at birth should minimize the duration of foetal exposure to maternal fluids and blood [28].

Slowik MK. et al. conducted a study on the presence of hepatitis $\mathrm{B}$ and $\mathrm{C}$ viruses in infants and young children. The diagnosis of chronic HBV is confirmed by positive serological tests on two occasions, at a distance of at least 6 months. The diagnosis of HCV in the new-born should be postponed until after the child reaches the age of 1 , as infants may have transient viremia [29].

The lack of an effective $\mathrm{HCV}$ vaccine (still under development) and the risk of mother-to-child transmission, increase the number of children with vertically acquired HCV. More effective and fewer toxic therapies are needed for young patients with $\mathrm{HBV}$ and $\mathrm{HCV}$, as well as methods of stopping perinatal HBV and HCV transmission.

HIV (Human Immunodeficiency Virus) is the etiological factor of acquired immunodeficiency syndrome (AIDS). The HIV virus infects cells of the immune system, especially CD4 + T lymphocytes or T helper lymphocytes, being able to synthesize its own genetic information in a form that can be incorporated into the genome of the host cell. Here it persists in a latent state or causes the destruction of cells following the replication of viral particles that will infect other CD4 + lymphocytes, causing the gradual and progressive destruction of the host's immune system. Patients are contagious through blood, sexual secretions, transplacental and breast milk. The number of CD4 cells should be assessed at least every three months during pregnancy. For patients who have received combination antiretroviral therapy (ART) with consistent viral suppression and CD4 counts above the threshold for opportunistic infections, monitoring of CD4 cell counts may be performed every six months [30,31]

Determining the exact gestational age in the fetus is especially important for HIV-positive pregnant women, as it may be necessary to terminate the pregnancy prematurely to reduce the risk of transmitting HIV infection. It is recommended to give birth by caesarean section at 38 weeks of gestation in case of viremia > 1000 copies / ml [32].

The earliest possible association of ART, together with the choice of birth by scheduled caesarean section and the complete lack of breastfeeding determine the expected neonatal results, the risk of vertical transmission decreasing up to $1-2 \%$.

HIV infection in children is most often caused by mother-to-child transmission. Sturt A.S. et al. analysed the 3 means of maternal-foetal transmission: during pregnancy, during birth and breastfeeding. They associated antiretroviral therapy in these patients and evaluated its effectiveness in reducing the rate of foetal illness. They 
demonstrated that the association of ART with caesarean section before the onset of labour and the total lack of breastfeeding reduced the risk of maternal-foetal transmission up to $1-2 \%$ [33].

Congenital rubella - its major impact occurs during pregnancy, when it can have devastating effects on the developing fetus. If the primary infection occurs as close as possible to birth, Rubella can cause a mild, self-limiting disease.

Congenital rubella syndrome (CRS) may include growth retardation, hepatosplenomegaly, thrombocytopenia, purpura, hyperbilirubinemia, deafness, cataracts, heart malformations, neurological and endocrinological sequelae.

Important cardiac abnormalities include pulmonary artery stenosis, aortic or pulmonary valve stenosis, ventricular septal defect (DSV), aortic coarctation, Fallot tetralogy. When diagnosed antepartum, it directs the obstetrician in choosing the way to terminate the pregnancy, in order to reduce the aggravation of the foetal pathology.

An update of cardiovascular malformations in congenital rubella syndrome was performed by Oster M.E. et al. They analysed 10 studies and identified 121 patients with a history of catheterization. $78 \%$ of them had stenosis of the pulmonary artery, and $62 \%$ had atrial canal persistence (PDA). In $49 \%$ of cases, both pulmonary artery stenosis and PDA were present, while isolated pulmonary artery stenosis and isolated PDA were found in $29 \%$ and $13 \%$ of cases, respectively. Of the 12 patients in the 10 studies with echocardiographic data, PDA was more common than pulmonary artery stenosis, but this finding is very limited by the small number of patients and echocardiography performed. Although published studies on cardiovascular malformations (CVM) in congenital rubella syndrome (CRS) have reported that PDA is the most commonly associated cardiovascular malformation with CRS, of the cases of CRS assessed by catheterization, pulmonary artery stenosis was actually more common than PDA. The combination of pulmonary artery stenosis and PDA was more common than branched pulmonary artery stenosis or PDA alone. Isolated pulmonary artery stenosis was twice as common as isolated PDA [34].

Congenital cytomegalovirus - Cytomegalovirus (CMV) infection is the leading cause of non-hereditary neurosensory deafness (SNHL), being detected in an asthma in new-borns with asymptomatic disease [35,36].

At birth, only about $10 \%$ have symptoms, with clinical manifestations like rush, neonatal jaundice, hepatosplenomegaly, thrombocytopenia, small birth weight for gestational age ("small for age"), microcephaly, intracranial calcifications, chorioretinitis, convulsions [37].
In a prospective multicentre study involving 539 very low birth weight infants (VLBW), the cumulative incidence of postnatal CMV infection at 12 weeks postnatal was $7 \%$. Transmission occurred from CMVpositive breast milk in almost all cases $(96 \%)$ and there were no transfusion-related infections. 5 out of 29 (17\%) infants infected with CMV developed symptomatic CMV disease, and three infants (10\%) died [38].

Herpes simplex virus - herpes simplex virus (HSV) infection during pregnancy poses an increased risk to the developing fetus and the new-born.

Most cases of neonatal HSV infection are acquired perinatal. HSV is transmitted to the baby during birth, mainly through the infected birth canal. The risk of transmission is higher when the primary HSV infection occurs during pregnancy [39]. Although many new-borns with HSV are born prematurely, most appear normal at birth. The infection is manifested by rash on the skin, eyes or mouth (SEM - "Skin, Eye, Mouth"), central nervous system (CNS) damage or disseminated disease involving several organs.

The initial manifestations of CNS disease are often nonspecific and include temperature variations (fever/ subfebrile), respiratory stress, lethargy and inadequate nutrition. They can progress fairly quickly to hypotension, jaundice, disseminated intravascular coagulation (CID), apnoea, and shock. Lesions (herpetiform vesicles) of the skin may or may not be present and can develop late. The absence of skin lesions complicates the recognition of the infection [39].

Brown Z.A. et al. studied the rate of neonatal HSV infection. They analysed 58,362 pregnant women who tested positive for HSV. Of these, 202 women were diagnosed with HSV during labour and only 10 (5\%) transmitted the disease to the new-born. Caesarean delivery significantly reduced the rate of HSV transmission among women diagnosed with HSV ( 1 in 85 caesareans vs. 9 in 117 vaginal) [39].

\section{Conclusions}

No teratogenic agent should be described qualitatively as a teratogenic, as a teratogenic exposure includes not only the agent, but also the dose and trimester of pregnancy when the exposure should occur.

When assessing the risk of exposure, the dose is a crucial component in determining the risk.

Teratogens follow a dose-response toxicological curve. This means that each teratogen has a threshold dose below which there is no risk of teratogenesis, regardless of when the exposure occurred during pregnancy.

It is easier to exclude an agent as the cause of birth defects than to conclude definitively that it is responsible for them, due to the existence of genocopies of teratogenic syndromes. 
The administration of drugs before and especially during pregnancy must be carried out carefully, with the knowledge of their potential teratogenic effects.

Most foetal malformations have a genetic component.

Infections associated with pregnancy cause an unfavorable prognosis on it, causing abortion, premature birth, foetal malformations or even foetal death.

Congenital structural defects are affected by a number of related environmental risk factors.

All pregnant women should be tested for syphilis as early as possible during pregnancy to prevent mother-tochild transmission.

Foetal chickenpox syndrome is a serious and rare condition in the new-born that can be prevented by vaccinating the mother before pregnancy.

The transmission of HIV infection from mother to fetus is dependent on the time of exposure of the fetus to maternal secretions.

Congenital rubella syndrome is associated with significant cardiovascular malformations.

Rates of neonatal HSV infection may be reduced by caesarean delivery and by limited vaginal examination during labor.

In the case of pregnant women with CMV infection, the main source of transmission is postnatal, through breast milk.

\section{Conflict of interest disclosure}

There are no known conflicts of interest in the publication of this article. The manuscript was read and approved by all authors.

\section{Compliance with ethical standards}

Any aspect of the work covered in this manuscript has been conducted with the ethical approval of all relevant bodies and that such approvals are acknowledged within the manuscript.

\section{Abbreviations}
AIDS - Acquired immunodeficiency syndrome
ART - Combined antiretroviral therapy
CID - Disseminated intravascular coagulation
CMV - Cytomegalovirus
CNS - Central nervous system
CRS - Congenital rubella syndrome
CVM - Cardiovascular malformations
CVS - Congenital chickenpox syndrome
DNA - Deoxyribonucleic acid
DSV - Ventricular septal defect
FB - Foetal blood
HBV - Hepatitis B virus

$\mathrm{HCV}$ - Hepatitis C virus

HIV - Human Immunodeficiency Virus

HSV - Herpes simplex virus

PCR - Polymerase chain reaction

PDA - Patent ductus arteriosus

SEM - "Skin, Eye, Mouth"

SNHL - Non-hereditary neurosensory deafness

TORCH- toxoplasma, other infection, rubella, cytomegalovirus, herpes simplex

VLBW - Very low-birthweight

VZV - Chickenpox virus

\section{References}

1. Calado AM, Dos Anjos Pires M. An Overview of Teratology. Methods Mol Biol. 2018;1797:3-32. doi:10.1007/978-1-4939-7883-0_1

2. Brent RL. Environmental causes of human congenital malformations: the pediatrician's role in dealing with these complex clinical problems caused by a multiplicity of environmental and genetic factors. Pediatrics. 2004;113(4 Suppl):957-968.

3. Chen G, Li C, Pei LJ, Zhang T, Zheng XY. [The relationships between exposure of periconceptional environmental risk factors and risks of common, structural birth defects]. Zhonghua Liu Xing Bing Xue Za Zhi. 2008 Mar;29(3):212-5.

4. Cullen A, Brown S, Cafferkey M, O'Brien N, Griffin E. Current use of the TORCH screen in the diagnosis of congenital infection. J Infect. 1998;36(2):185-188. doi:10.1016/s0163-4453(98)80011-X

5. Paquet C, Yudin MH; Society of Obstetricians and Gynaecologists of Canada. Toxoplasmosis in pregnancy: prevention, screening, and treatment. $J$ Obstet Gynaecol Can. 2013;35(1):78-81. doi:10.1016/s1701-2163(15)31053-7

6. McDonnell M, Cullen A, Kiberd B, Mehanni M, Matthews T. A national model of care service for professionals dealing with sudden infant death. Ir J Med Sci. 1999;168(4):237-241. doi: 10.1007/BF02944347

7. Newman L, Rowley J, Vander Hoorn S, et al. Global Estimates of the Prevalence and Incidence of Four Curable Sexually Transmitted Infections in 2012 Based on Systematic Review and Global Reporting. PLoS One. 2015;10(12): $\mathrm{e} 0143304$. doi:10.1371/journal.pone.0143304

8. Sparling PF. Natural history of syphilis. In: Sexually Transmitted Diseases, Holmes KK, Mardh PA, Sparling PF, et al (Eds), McGraw-Hill, New York 1990. pp. 213.

9. Cheng JQ, Zhou H, Hong FC, et al. Syphilis screening and intervention in 500,000 pregnant women in 
Shenzhen, the People's Republic of China. Sex Transm Infect. 2007;83(5):347-350.

doi:10.1136/sti.2006.023655

10. Lamont RF, Sobel JD, Carrington D, et al. Varicellazoster virus (chickenpox) infection in pregnancy. BJOG. 2011;118(10):1155-1162. doi:10.1111/j.14710528.2011.02983.x

11. Koren G. Congenital varicella syndrome in the third trimester. Lancet. 2005;366(9497):1591-1592. doi: 10.1016/S0140-6736(05)67643-X

12. Laforet EG, Lynch CL Jr. Multiple congenital defects following maternal varicella; report of a case. $N$ Engl J Med. 1947;236(15):534-537. doi:10.1056/NEJM194704102361504

13. Mazzella M, Arioni C, Bellini C, Allegri AE, Savioli C, Serra G. Severe hydrocephalus associated with congenital varicella syndrome. CMAJ. 2003;168(5): 561-563.

14. Mouly F, Mirlesse V, Méritet JF, et al. Prenatal diagnosis of foetal varicella-zoster virus infection with polymerase chain reaction of amniotic fluid in 107 cases. Am J Obstet Gynecol. 1997;177(4): 894-898. doi:10.1016/s0002-9378(97)70291-6

15. Cuthbertson G, Weiner CP, Giller RH, Grose C. Prenatal diagnosis of second-trimester congenital varicella syndrome by virus-specific immunoglobulin M. J Pediatr. 1987; 111(4): 592-595. doi: 10.1016/s0022-3476(87)80128-2

16. Isada NB, Paar DP, Johnson MP, et al. In utero diagnosis of congenital varicella zoster virus infection by chorionic villus sampling and polymerase chain reaction. Am J Obstet Gynecol. 1991;165(6 Pt 1):17271730. doi:10.1016/0002-9378(91)90023-k

17. Sanchez MA, Bello-Munoz JC, Cebrecos I, et al. The prevalence of congenital varicella syndrome after a maternal infection, but before 20 weeks of pregnancy: a prospective cohort study. J Matern Foetal Neonatal Med. 2011;24(2):341-347. doi: $10.3109 / 14767058.2010 .497567$

18. Sookoian S. Liver disease during pregnancy: acute viral hepatitis. Ann Hepatol. 2006;5(3):231-236.

19. Hieber JP, Dalton D, Shorey J, Combes B. Hepatitis and pregnancy. J Pediatr. 1977;91(4):545-549. doi: 10.1016/s0022-3476(77)80499-X

20. Williams R. Global challenges in liver disease. Hepatology. 2006;44(3):521-526.

doi:10.1002/hep. 21347

21. Schweitzer A, Horn J, Mikolajczyk RT, Krause G, Ott JJ. Estimations of worldwide prevalence of chronic hepatitis B virus infection: a systematic review of data published between 1965 and 2013. Lancet. 2015; 386(10003): 1546-1555. doi: 10.1016/S01406736(15)61412-X
22. Slowik MK, Jhaveri R. Hepatitis B and C viruses in infants and young children. Semin Pediatr Infect Dis. 2005; 16(4): 296-305. doi: 10.1053/j.spid.2005.06.009

23. Bortolotti F, Resti M, Giacchino R, et al. Changing epidemiologic pattern of chronic hepatitis $\mathrm{C}$ virus infection in Italian children. $J$ Pediatr. 1998;133(3):378-381.

doi:10.1016/s0022-3476(98)70273-2

24. Bortolotti F, Iorio R, Resti M, et al. An epidemiological survey of hepatitis $\mathrm{C}$ virus infection in Italian children in the decade 1990-1999. J Pediatr Gastroenterol Nutr. 2001;32(5): 562-566. doi:10.1097/00005176200105000-00013

25. Bortolotti F, Iorio R, Resti M, et al. Epidemiological profile of 806 Italian children with hepatitis $\mathrm{C}$ virus infection over a 15-year period. J Hepatol. 2007;46(5):783-790. doi: 10.1016/j.jhep.2006.12.014

26. Rowe CR, Newberry DM, Jnah AJ. Congenital Syphilis: A Discussion of Epidemiology, Diagnosis, Management, and Nurses' Role in Early Identification and Treatment. Adv Neonatal Care. 2018;18(6):438445 .

doi:10.1097/ANC.0000000000000534

27. Hughes BL, Page CM, Kuller JA. Society for MaternalFoetal Medicine (SMFM). Hepatitis C in pregnancy: screening, treatment, and management. Am J Obstet Gynecol. 2017;217(5): B2-B12. doi:10.1016/j.ajog.2017.07.039

28. Younes AS, Csire M, Kapusinszky B, Szomor K, Takács M, Berencsi G. Heterogeneous pathways of maternal-foetal transmission of human viruses (review). Pathol Oncol Res. 2009;15(3):451-465. doi:10.1007/s12253-009-9166-9

29. Hu X, Wang L, Xu F. Guides concerning tenofovir exposure via breastfeeding: A comparison of drug dosages by developmental stage. Int J Infect Dis. 2019;87:8-12. doi: 10.1016/j.ijid.2019.07.023

30. Swamy GK, Garcia-Putnam R. Maternal immunization to benefit the mother, fetus, and infant. Obstet Gynecol Clin North Am. 2014; 41(4): 521-534. doi: 10.1016/j.ogc.2014.08.001

31. Tuomala RE, Kalish LA, Zorilla C, et al. Changes in total, CD4+, and CD8+ lymphocytes during pregnancy and 1 year postpartum in human immunodeficiency virus-infected women. The Women and Infants Transmission Study. Obstet Gynecol. 1997;89(6):967974. doi: 10.1016/s0029-7844(97)00129-4

32. Gale HB, Gitterman SR, Hoffman HJ, et al. Is frequent CD4+ T-lymphocyte count monitoring necessary for persons with counts $>=300$ cells $/ \mu \mathrm{L}$ and HIV-1 suppression?. Clin Infect Dis. 2013;56(9):1340-1343. doi:10.1093/cid/cit004

33. Sturt AS, Dokubo EK, Sint TT. Antiretroviral therapy (ART) for treating HIV infection in ART-eligible 
pregnant women. Cochrane Database Syst Rev. 2010;(3):CD008440. Published 2010 Mar 17. doi:10.1002/14651858.CD008440

34. Oster ME, Riehle-Colarusso T, Correa A. An update on cardiovascular malformations in congenital rubella syndrome. Birth Defects Res A Clin Mol Teratol. 2010;88(1):1-8. doi: 10.1002/bdra.20621

35. Harrison GJ. Cytomegalovirus. In: Feigin and Cherry's Textbook of Pediatric Infectious Diseases, 7th ed, Cherry JD, Harrison GJ, Kaplan SL, et al (Eds), Elsevier Saunders, Philadelphia 2014. pp. 1969.

36. Goderis J, De Leenheer E, Smets K, Van Hoecke H, Keymeulen A, Dhooge I. Hearing loss and congenital CMV infection: a systematic review. Pediatrics. 2014;134(5):972-982. doi:10.1542/peds.2014-1173
37. Thorne C, Newell ML. Safety of agents used to prevent mother-to-child transmission of HIV: is there any cause for concern?. Drug Saf. 2007;30(3):203-213. doi:10.2165/00002018-200730030-00004

38. Josephson CD, Caliendo AM, Easley KA, et al. Blood transfusion and breast milk transmission of cytomegalovirus in very low-birth-weight infants: a prospective cohort study. JAMA Pediatr. 2014;168(11):1054-1062.

doi:10.1001/jamapediatrics.2014.1360

39. Brown ZA, Wald A, Morrow RA, Selke S, Zeh J, Corey L. Effect of serologic status and cesarean delivery on transmission rates of herpes simplex virus from mother to infant. JAMA. 2003;289(2):203-209. doi: 10.1001/jama.289.2.203 